Article

\title{
The Effect of Annulation Upon the Solvatochromic Behaviour of Related Merocyanines
}

\author{
Marcos Caroli Rezende \\ Facultad de Química y Biología, Universidad de Santiago de Chile, Casilla 40, Correo \\ 33, Santiago,Chile; e-mail: mcaroli@lauca.usach.cl
}

Received: May 13, 1997

\begin{abstract}
O efeito da anelação em fragmentos aromáticos doadores e/ou aceptores em merocianinas aparentadas é discutido com auxílio de um modelo teórico baseado em cálculos semi-empíricos utilizando o método AM1. As previsões teóricas são validadas com exemplos de oito corantes solvatocrômicos descritos na literatura.

The effect of annulation of the donor and/or the acceptor ring fragments of related merocyanines is discussed with the aid of a theoretical model based on semiempirical calculations performed with the AM1 method. The theoretical expectations are validated with examples of eight solvatochromic dyes described in the literature.
\end{abstract}

Keywords: solvatochromic merocyanines, annulation, semiempirical calculations

\section{Introduction}

The influence of solvents on the electronic spectra of solvatochromic dyes has been a subject of much interest in the past decades. According to their spectral behaviour with the changing polarity of the medium, dyes are classified as exhibiting positive or negative solvatochromism. The former behaviour, when the longest wavelength band in the visible is bathochromically shifted with the increasing polarity of the medium, is observed with those dyes whose ground state is less polar than the excited state. Conversely, dyes which exhibit negative solvatochromism have their longest wavelength band shifted to shorter $\lambda$ values when the solvent polarity is increased, an effect which is rationalized as arising from a more polar ground state than the excited state. Some dyes may also change their solvatochromic behaviour at a given polarity value, a phenomenon which is known as solvatochromic reversal.

The design of new solvatochromic dyes requires a proper understanding of their behaviour in solution, and how specific structural features affect their spectral properties in different media. For this reason, parallel to synthesizing new dyes, researchers have been concerned with explaining and predicting their spectral variations in solution by means of theoretical models. This has not been an easy task because experimental transition energies reflect not only non-specific effects, which are amenable to a theoretical treatment that regards the solvent as a continuum, but are also the result of specific dye-solvent interactions, which are in a sense unique for each solvatochromic dye. The problem has been tackled in different ways. A first approach takes into account specific dye-solvent interactions, and strives to reproduce experimental transitions by combining a continuum model with a supramolecular treatment of such interactions. Alternatively, one may ignore specific dye-solvent interactions to arrive at a description which agrees qualitatively with the experimental solvatochromic behaviour of a dye.

An example of the first approach is the rationalization of the negative solvatochromic behaviour of the $\mathrm{E}_{\mathrm{T}}$ (30) dye by employing the AM1 Hamiltonian of Dewar in the geometry optimizations and the spectroscopy designed INDO/S method ${ }^{1}$. In trying to relate calculated transition energies with absorptions in specific solvents, the authors combined a continuum model, to deal with non-specific medium effects, with calculations on dye-solvent arrangements, in a supramolecular fashion. The latter treatment accounted for specific effects such as hydrogen bonds, and obviously varied from solvent to solvent.

Ignoring such specific interactions leads to transition energies which generally depart largely from experimental values. However, by emphasizing trends, instead of par- 
ticular values, this second approach provides a means of comparing the solvatochromic behaviour of related dyes. The experimental chemist may thus not only rationalize such trends, but also correlate them with structural features and design new compounds with anticipated properties.

We have adopted this second approach in the study of the solvatochromic behaviour of merocyanines. Our approach was based on the influence of an external electric field upon the geometry and electronic levels of a given $\mathrm{dye}^{2}$. It was also related to other proposals in the literature for the treatment of the spectra ${ }^{3}$, polarizabilities and hyperpolarizabilities of merocyanines ${ }^{4}$. An approach similar to ours was followed in a study of the effect of a variable electric field on the frontier orbitals of a symmetrical bipyrazine-1,6-hexatriene dication ${ }^{5}$. However, contrary to other treatments which tried to interpret the solvatochromic behaviour of a given dye by calculation of its properties in a few different media only ${ }^{3,5,6}$, we emphasized the need of calculating its properties in several environments of changing polarities. Though based on a simplified theoretical model, the results of such calculations have been successfully compared with qualitative trends exhibited by a family of related pyridinium phenoxides ${ }^{2}$, and in comparing the solvatochromic behaviour of a previously described vinylogous $\gamma$-pyridone with its $\alpha$-analogue synthesized by us ${ }^{7}$.

In the present paper we investigate the effect of annulation of a donor and/or acceptor fragment upon the solvatochromism of related merocyanines. Calculations on simple models lead to patterns of solvatochromic behaviour which are validated by comparison with examples taken from the literature. Thus, a simple theoretical model may be used in designing new dyes with a predictable solvatochromic behaviour.

\section{Experimental}

Geometry optimizations were carried out utilizing the AM1 semi-empirical method of the MOPAC 6.0 package $^{8}$, with the only restraint of constant planarity of the aromatic rings.

Environmental polarity variations were mimicked by positioning a +2 sparkle at a variable distance from the oxygen atom of each phenoxide dye in its most stable conformation. We thus obtained a variable $\mathrm{C}=\mathrm{O}$ bond distance for each dye, as a function of the sparkle-oxygen distance. This $\mathrm{C}=\mathrm{O}$ bond distance was employed for the definition of our normalized "polarity" scale P. P values were obtained from the relationship $P=\left(d-d_{n}\right) /\left(d_{p}-d_{n}\right)$. Here, $\mathrm{d}$ was the $\mathrm{C}=\mathrm{O}$ bond distance at a given dye-sparkle separation, $\mathrm{d}_{\mathrm{n}}$ was this bond distance in a totally non-polar environment calculated by geometry optimization of the dye structure in the absence of any sparkle, and $d_{p}$ was this distance in the polar extreme of the scale, calculated with a dye-sparkle distance of $2 \AA$.

\section{Results and Discussion}

The merocyanines discussed in this work comprise a family of dyes with aromatic donor and acceptor fragments, optionally joined by a conjugated bridge of a variable number of vinylic units. The donor is a phenoxide group, or its derivative, and the acceptor is a pyridinium ring derivative.

The structure of these compounds can be described as a hybrid of two canonical forms, a non-polar (I) and a zwitterionic formula (II).

The reverse solvatochromism of many of these dyes is rationalized by structural changes of the molecule in the ground and excited states, as the polarity of the medium is altered ${ }^{9}$.This is confirmed by the pmr spectra of these compounds in different solvents, which point to significant structural changes as the solvent polarity is increased ${ }^{10-12}$.

The effect of annulation upon the solvatochromism of related merocyanines was investigated with model systems $\mathbf{1 a}$ and $\mathbf{1 b}$, both of which were compared with system $\mathbf{1}$.

The solvatochromic behaviour of pyridinium phenoxide dyes reflects to a major extent the microenvironment around the phenoxide moiety of the molecule ${ }^{13}$, the $\mathrm{E}_{\mathrm{T}}(30)$ dye being one of the most conspicuous examples of this.

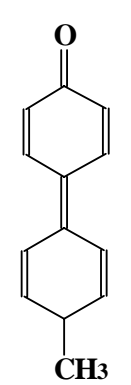

1

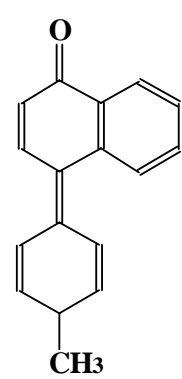

$1 \mathrm{a}$<smiles>CC1C=CC(=C2C=CC(=O)C=C2)c2ccccc21</smiles>

1b
Scheme 2.

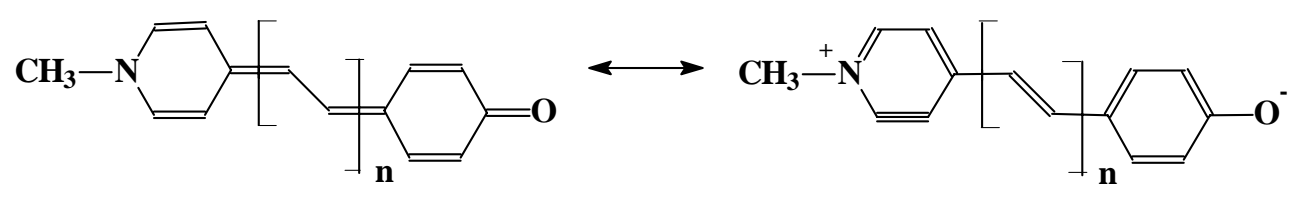

(I)

Scheme 1. 
Therefore, in mimicking polarity changes around systems like $\mathbf{1}$, we simply positioned a "sparkle" of charge +2 along the $\mathrm{C}-\mathrm{O}$ bond of the phenoxide ring, at a variable distance from the oxygen atom. The resulting variable electric field induced geometrical and electronic perturbations upon the system, so that, to each given "polarity" of the medium corresponded a given geometry and a HOMO-LUMO energy gap of the molecule. We chose the $\mathrm{C}-\mathrm{O}$ bond distance as a measure of the medium polarity. This distance evolved from a value near 1.22 angstroms in the absence of any sparkle, to a value of $c a .1 .32$ angstroms in the polar environment generated by the sparkle 2 angstroms away from the oxygen atom.

We next equaled the charge-transfer-transition energy of the dye in each medium to its HOMO-LUMO energy gap. This approximation was supported by the observation that the monoelectronic HOMO-LUMO transition is the major contributor to calculated absorption energies when configuration interactions are incorporated into a more rigorous calculation ${ }^{3,5,14}$.

By plotting the calculated energy gap $\Delta \mathrm{E}$ against the normalized polarity values $P$ based on the variations of the $\mathrm{C}-\mathrm{O}$ bond distance of systems $\mathbf{1}$, as described in the Experimental, we obtained theoretical curves for the solvatochromic behaviour of these model dyes. These curves are shown in Fig. 1a. Inspection of these plots shows that increasing annulation of the donor phenoxide ring or of the acceptor pyridinium ring (Fig. 1b) has the same effect of decreasing the transition energies of the longest wave-

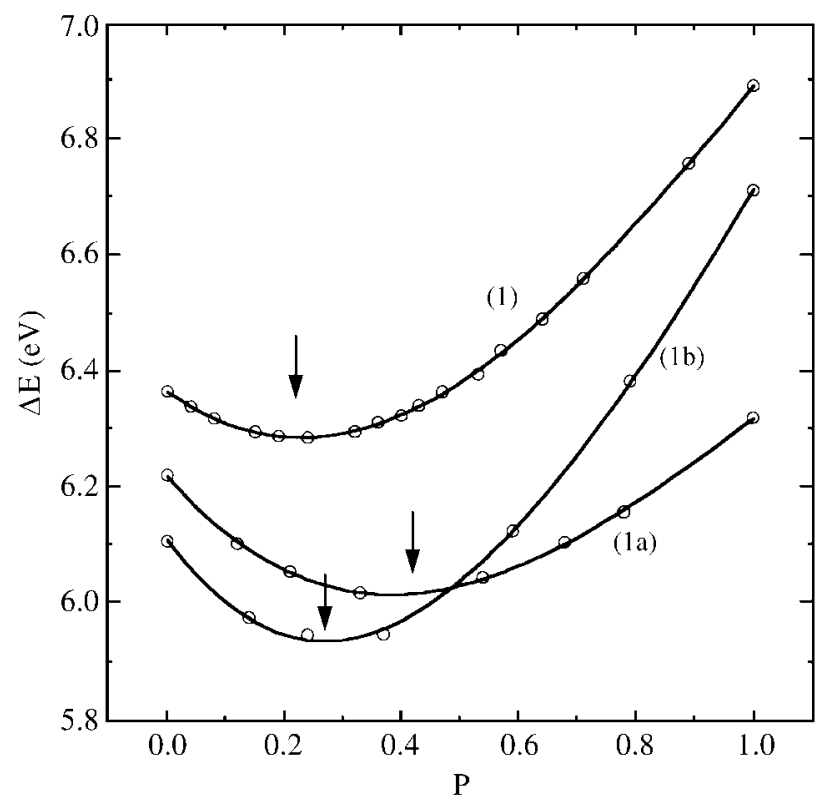

Figure 1. Calculated variation of the HOMO-LUMO energy gap $\Delta \mathrm{E}$ with the "polarity" $\mathrm{P}$ of the medium, for systems 1, 1a and $1 \mathrm{~b}$. Arrows indicate the points of solvatochromic reversal for the structures. length band (smaller wavenumbers, larger $\lambda_{\max }$ values). In terms of the HSAB theory ${ }^{15,16}$, annulation of the donor or acceptor ring decreases the HOMO-LUMO gap and thus softens the resulting dye. This energy-gap variation with annulation is accompanied by a shift of the theoretical curve minimum to a more polar region, as can be seen in the Figures. We may thus expect that appropriate annulation of a negatively solvatochromic dye may eventually change it into a dye exhibiting solvatochromic reversal and even positive solvatochromism.

Compounds 2, 3, and 4 were described recently ${ }^{12}$ and constitute an example of a family of merocyanines where increasing annulation of the donor phenoxide and the acceptor pyridinium moieties leads to changes in their solvatochromic behaviour. In agreement with our expectations, the curve minimum gradually shifts to more polar regions as annulation $(\mathbf{2}<\mathbf{3}<\mathbf{4})$ increases (Fig. 2). Note that, while merocyanine $\mathbf{2}$ exhibits only negative solvatochromism, dyes $\mathbf{3}$ and $\mathbf{4}$ present a solvatochromic reversal in solvents with $\mathrm{E}_{\mathrm{T}} \mathrm{N}$ values around 0.30 and 0.40 , respectively.

Another example of the effect of annulation upon the solvatochromism of two related dyes is given by the pair $\mathbf{5}$ and 6.

Compound $\mathbf{5}$ was reported by Gibson and Bailey as a negatively solvatochromic dye in the range of employed solvents ${ }^{17}$. The quinolizinium dye 6 was described more recently ${ }^{18}$ as exhibiting reverse solvatochromism in media of low polarity $\left(\mathrm{E}_{\mathrm{T}} \mathrm{N}=0.36\right)$. Both merocyanines can be

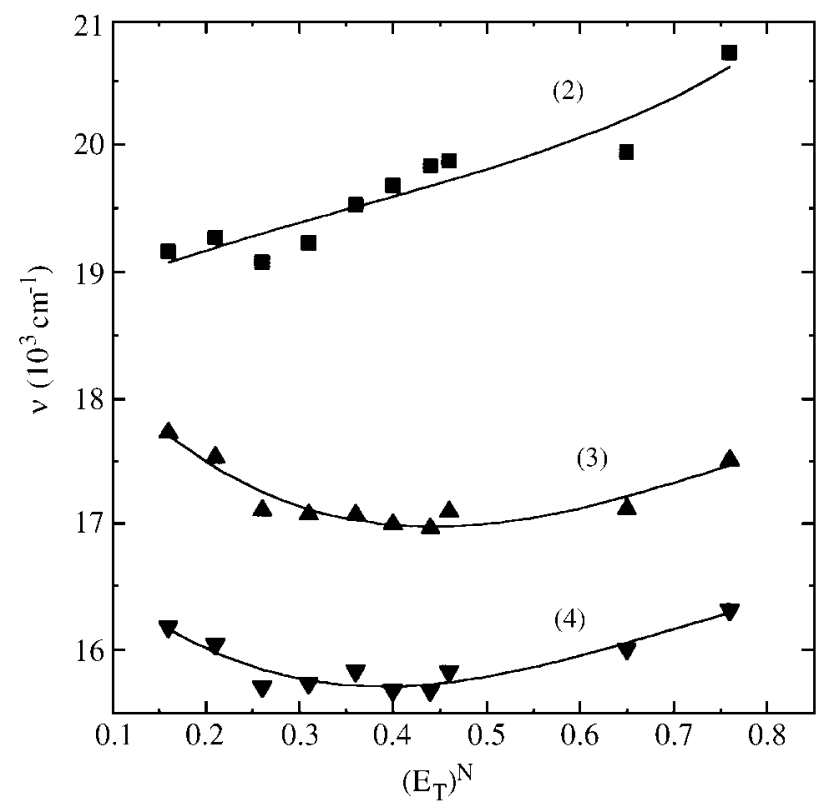

Figure 2. Variation of the position of the charge-transfer band, in wavenumber units, with the solvent polarity, expressed in the normalized $\mathrm{ET}_{\mathrm{T}}{ }^{\mathrm{N}}$ scale, for dyes 2, 3 and 4. Solvents range from non-polar (v.g. $\mathrm{CHCl}_{3}$, $\mathrm{CH}_{2} \mathrm{Cl}_{2}$, THF) and polar aprotic (DMF, DMSO) to polar protic (MeOH, EtOH). Data were taken from Ref. 12. 
regarded as vinylogous $\gamma$-pyridones which differ in the degree of annulation of the acceptor pyridinium/quinolizinium. The increased annulation found in $\mathbf{6}$ should render this dye softer than $\mathbf{5}$. In addition, it should shift the theoretical curve minimum to the right, and eventually lead to an observable solvatochromic reversal of the quinolizinium dye, as the medium polarity is varied. The experimental data, shown in Fig. 3, support both expectations.

Annulation of the donor and/or acceptor fragments is thus seen to affect both the HOMO-LUMO gap of a dye, and its response to polarity variations of the solvent. One last example of this is provided by dyes 7, 8, and 9 .

Dye 7 was described by Buncel et al. ${ }^{19}$ as exhibiting negative solvatochromism. A plot of its absorption wavenumber in different solvents, $v_{\max }$,against the corresponding $\mathrm{E}_{\mathrm{T}}$ polarity values yielded a straight line with a

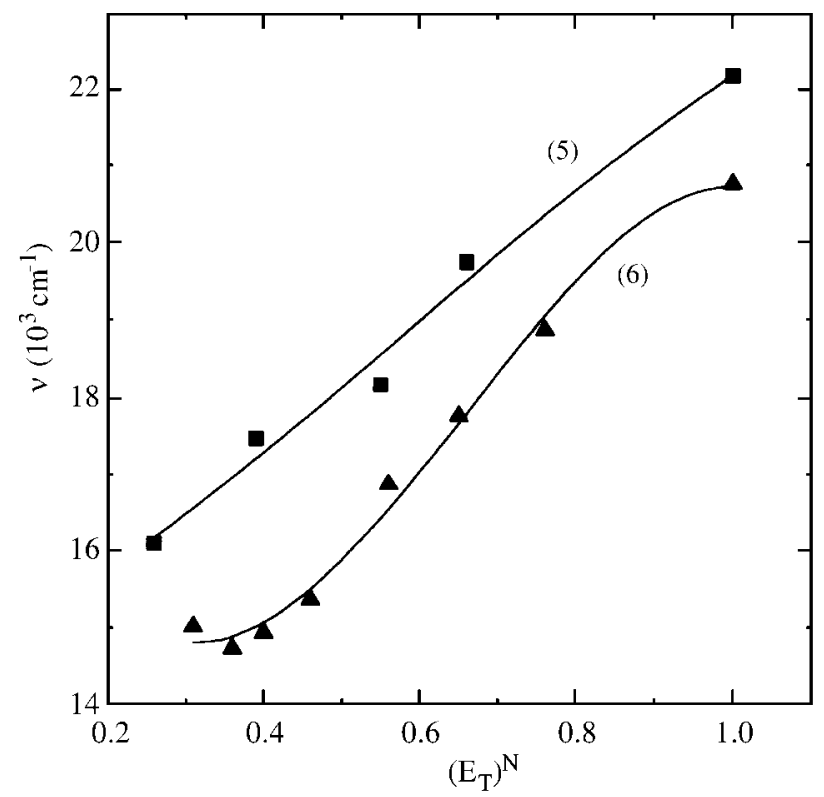

Figure 3. Variation of the position of the charge-transfer band, in wavenumber units, with the solvent polarity, expressed in the normalized $\mathrm{ET}^{\mathrm{N}}$ scale, for dyes 5 and 6 . Solvents range from non-polar $\left(\mathrm{CHCl}_{3}\right)$ and polar aprotic (DMF, MeCN) to polar protic (alcohols and water). Data were taken from Refs. 17 and 18.<smiles>CC(C)CC1=CC(=C2C=CN(C)C=C2)C=C(CC(C)C)C1=O</smiles>

2<smiles>CCC(C)C1=CC(=C2C=CN(C)c3ccccc32)C=C(CC(C)C)C1=O</smiles>

3<smiles>CCC(C)C1=CC(=C2C=CN(C)c3ccccc32)c2ccccc2C1=O</smiles>

4

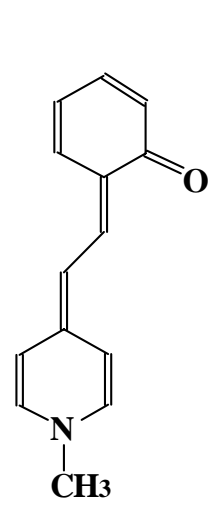

5

5

positive slope, $v_{\max }=12800+113 \mathrm{E}_{\mathrm{T}}$. By contrast, compounds 8 and 9 were both positively solvatochromic dyes. Linear regression of the experimental points yielded straight lines with negative slopes, $v_{\max }=17880-27.1 \mathrm{E}_{\mathrm{T}}$, for compound 8, and $v_{\max }=18600-34.1 \mathrm{E}_{\mathrm{T}}$, for compound $\mathbf{9}^{20}$. The causes for such a discrepancy remained seemingly obscure to the authors, the absence of solute-solvent interactions in the calculations being invoked as a possible explanation for this disagreement.

In the light of our discussion, however, these discrepancies are not surprising. Systems 7, 8, and 9 differ not only<smiles>O=C1C=CC=CC1=CC=C1C=CN2C(=C1)C=Cc1ccccc12</smiles>

6

Scheme 4.<smiles>CN1/C(=C\C=C2C=C([N+](=O)[O-])C=CC2=O)C(C)(C)c2ccccc21</smiles>

7<smiles>CN1/C(=C\N=C2C(=O)C=Cc3ccccc32)C(C)(C)c2ccccc21</smiles><smiles>CN1/C(=C\N=C2C(=O)c3ccccc3-c3ccccc32)C(C)(C)c2ccccc21</smiles>

9

Scheme 3.

Scheme 5. 
in the degree of annulation of their phenoxide moiety. The unsaturated link of the donor/acceptor fragments of $\mathbf{8}$ and 9 exhibits a nitrogen atom, absent in system $\mathbf{7}$. In addition, a nitro substituent in $\mathbf{7}$ is absent in $\mathbf{8}$ and $\mathbf{9}$.

The replacement of a methine by a nitrogen atom in the unsaturated link of these systems cannot be held responsible for their differences in solvatochromic behaviour. Theoretical considerations predict that such replacements, in an "even" or "unstarred" position of a polymethine chain, should not lead to any qualitative changes in the solvatochromic behaviour of the dye $\mathrm{e}^{21}$. This prediction has been verified experimentally by the behaviour of some iminomerocyanines synthesized by us ${ }^{22}$. As for the nitro substituent in 7, it should stabilize a zwitterionic canonical formula of the dye hybrid, in an opposite effect to that of increasing annulation of the donor phenoxide. As a result, the suppression of a nitro substituent on the aromatic donor group adds to the effect of the increased annulation in compounds 8 and 9 . The result is a complete change of the solvatochromic behaviour of dye 7. The latter compound, a negatively solvatochromic merocyanine is thus seen to evolve into a positively solvatochromic dye as a result of appropriate changes of its structure.

In conclusion, the above examples illustrate the use of a simple model for the qualitative prediction of the solvatochromic behaviour of related merocyanines. Measuring the electronic perturbations induced by point charges brought gradually closer to a dye structure may give a reasonable idea of its solvatochromic behaviour. Though the model may be refined by the incorporation of configuration interactions into the calculations ${ }^{7}$, qualitative conclusions regarding the effect of specific structural features in a series of dyes may do without such refinements. Thus, for a family of pyridinium phenoxide dyes, increasing annulation of the pyridinium acceptor or the phenoxide donor ring should lead to softer dyes, which tend to absorb at longer wavelengths. This should be accompanied by behaviourial changes: thus, upon increasing annulation, a negatively solvatochromic dye may eventually show a reverse solvatochromic behaviour and even become a purely positively solvatochromic dye. These predictions are again born out by data from the literature.

\section{Acknowledgment}

Support from the Directoría de Investigaciones Científicas y Tecnológicas (DICYT) of the Universidad de Santiago de Chile is gratefully acknowledged.

\section{References}

1. Alencastro, R.B.; Neto, J.D.M.; Zerner, M.C. Int. J. Quant. Chem. 1994, 28, 361.

2. Silva, L.; Machado, C.; Rezende, M.C. J. Chem. Soc. Perkin Trans. 2 1995, 483.

3. Morley, J.O. J. Mol. Struct. (Theochem.) 1994, 304, 191.

4. Marder, S.R.; Gorman, C.B.; Meyers, F.; Perry, J.W.; Bourhill, G.; Brédas, J.-L.; Pierce, B.M Science 1994, 265,632 .

5.Eriksson, L.A.; Benachenhou, N.S.; Goscinski, O. Molecular Engineering 1995, 4, 339.

6. Luzhkov, V.; Warshel, A. J. Am. Chem. Soc. 1991, 113, 4491.

7. Aliaga, C.; Galdames, J.S.; Rezende, M.C. J. Chem. Soc. Perkin Trans 2 1997, 1055.

8. MOPAC 6.0, Quantum Chemistry Exchange Program, University of Bloomington, Indiana.

9. Reichardt, C. Solvents and Solvent Effects in Organic Chemistry 2nd ed.., VCH, Weinheim, 1988, p. 298.

10.Pozzo, J.L.; Samat, A.; Guglielmetti, R.; DeKeukeleire, D. J. Chem. Soc. Perkin 2 1993, 1327.

11.Zaichenko, N.L.; V.Lyubimov, A.; Marevtsev, V.S.; Cherkashin, M.I. Transl. from Izv. Akad. Nauk, Ser. Khim. 1989, 1040.

12. Runser, C.; Fort, A.; Barzoukas, M.; Combellas, C.; Suba, C.; Thiébault, A.; Graff, R.; Kintzinger, J.P. Chem. Physics 1995, 193, 309.

13.Zanotto, S.P.; Scremin, M.; Machado, C.; Rezende, M.C. J. Phys. Org. Chem. 1993, 6, 637.

14. Botrel, A.; LeBeuze, A.; Jacques, P.; Strub, H. J. Chem. Soc. Faraday Trans. 2 1984, 80, 1235.

15. Pearson, R.G. J. Am. Chem. Soc. 1985, 107, 6801.

16. Pearson, R.G. J. Chem. Educ. 1987, 64, 561.

17. Gibson, H.W.; Bailey, F.C. Can. J. Chem. 1975, 53, 2162.

18. Arai, S.; Arai, H.; Hida, M.; Yamagishi, T. Heterocycles 1994, 38, 2449.

19. Keum, S.R.; Hur, M.S.; Kazmaier, P.M.; Buncel, E. Can. J. Chem. 1991, 69, 1940.

20. Favaro, G.; Masetti, F.; Mazzucato, U.; Ottavi, G., Allegrini, P.; Malatesta, V. J. Chem. Soc. Faraday Trans. 1994, 90, 333.

21. Buncel, E.; Rajagopal, S. Acc. Chem. Res. 1990, 23, 226.

22. Machado, C.; Nascimento, M.G.; Rezende, M.C. J. Chem. Soc. Perkin Trans. 2 1994, 2539. 